\title{
SLCT Inhibitor GNS561
}

National Cancer Institute

\section{Source}

National Cancer Institute. SLCT Inhibitor GNS561. NCI Thesaurus. Code C154553.

An orally available, quinolone-derived, small molecule inhibitor of an as of yet not disclosed solute carrier transporter (SLCT), with potential antineoplastic activity. Upon oral administration, GNS561 demonstrates multiple cellular effects including inhibition of SLCT activity, induction of apoptosis via caspase 3/7 activation, and inhibition of autophagy through lysosomal disruption. Several SLCTs are upregulated in cancer and serve as tumor promoters. Over-expression of SLCT in some tumors is associated with stemness features and may be associated with poor outcomes. Inhibition of autophagy and induction of apoptosis may potentially inhibit tumor cell growth. 